Original Research Paper

\title{
Testing VaR Accuracy for CDS Portfolios Using Historical Simulation and Delta-Normal Models
}

\author{
Viviane Y. Naimy, PhD \\ Professor of Finance, Department of Accounting and Finance, \\ Faculty of Business Administration and Economics, Notre Dame University, Louaize, Lebanon
}

\author{
Article history \\ Received: 16-02-2016 \\ Revised: 06-04-2016 \\ Accepted: 07-04-2016 \\ Email: vnaimy@ndu.edu.lb, \\ vnaimy@gmail.com
}

\begin{abstract}
This paper studies the accuracy of the VaR using the DeltaNormal and Historical approaches in measuring the risk of CDS portfolios in three different zones, US, Europe and Asia, for the period March 2013November 2015. The portfolios consist exclusively of CDS of high rated banks and financial institutions. We found that at the $95 \%$ confidence level both approaches were accurate using equal weights over 500 days. However, at the $99 \%$ level, the Delta-Normal method underestimated the VaR except for Aisa portfolio even with the use of exponential weights for different values of $\lambda$. The Historical Simulation approach was more accurate because it accounted for fat tails and therefore avoided nonstationarity. Both approaches failed in accurately measuring the VaR over 50 days.
\end{abstract}

Keywords: CDS, Historical Simulation, Delta-Normal Approach, Value at Risk

JEL Classification, C12, C22, C32

\section{Introduction}

Credit Default Swaps (CDS) are derivative instruments designed to manage credit risks efficiently. The advantage of CDSs over other credit derivatives is that the way they work is straightforward. A CDS is an instrument that provides insurance against the risk of a default by a particular company. The company is known as the reference entity and a default by the company is known as a credit event. They are agreements between a buyer and a seller, where the buyer pays to the seller a series of premiums until the end of the life of the CDS or until a credit event occurs and the seller agrees to buy the bonds for their face value when a credit event occurs such as corporate restructuring, bankruptcy and lower credit rating. CDS are derivatives which transfers the credit risk (underlying asset) from the buyer to the seller through swaps.

JPMorgan was the first to trade CDS in 1995. Then, CDS trading has considerably grown in the over-thecounter derivatives markets. The International Securities Dealer Association (ISDA) started collecting data on CDS in 2001. Total outstanding notional attained its peak in 2007 and exceeded USD 62 trillion. During the credit crisis of 2007, regulators became concerned that CDS were a source of systematic risk. That was mainly due to the losses experienced by the insurance company, AIG, the big seller of protection on the AAA-rated tranches created from mortgages. In 2009, this number was divided in two through portfolio compression due to the regulators requirements. Long and short credit derivative positions on the same underlying credit entity held by the same institution are netted (Duffie et al., 2010).

During the European sovereign debt crisis speculative activity in CDS markets intensified the debt problems of countries such as Greece which faced the biggest sovereign default the international markets have ever seen, resulting in an expected CDS payout of approximately USD 2.6 billion. Since that time CDS continued to trade actively with increased spreads and gained an important place in the derivatives markets. Therefore assessing the risk of CDS becomes a need for financial institutions, nonfinancial institutions, regulators and asset managers. The purpose of this paper is to use the Value at Risk (VaR) to assess and compare the CDS portfolios risks of some highly rated financial institutions in the US, Europe and Asia for the period 2013-2015.

Prior to the mid-1990, VaR was used by financial institutions. The VaR mathematics were developed in the context of portfolio theory where the focus on market risks contributed to how VaR is computed. Its usage was mainly due to the failure of the risk tracking process. Practically, the VaR can be used by any entity to measure its risk exposure and capture the potential loss in value due to adverse market movements over a 
specified period. VAR has been applied to market, credit and operational risk. In fact, linear sensitivity measures do not translate into a dollar loss and are called duration for exposure to interest rates, beta for exposure to stockmarket movements and delta for exposure of options to the underlying asset price and are used for setting limits. VaR combines the price-yield relationship with the probability of an adverse market movement. It is a statistical risk measure of potential losses. It also covers many other sources of risks such as commodities, equities, foreign currencies while considering leverage, correlations and current positions which are indispensable when dealing with large portfolios with derivatives.

VaR summarizes the worst loss over a target horizon that will not be exceeded with a given level of confidence. In other words, VaR answers what could happen under changes in market values. Practically, the VaR improved worldwide standards for managing many types of risk and is used by any institution exposed to financial risk through passive, defensive and active methods. VaR passively translates the financial risk level to shareholders in easy and straight forward terms. It is used defensively to control risk through setting position limits for traders. It is also used actively to manage the risk through the allocation of capital across the whole institution's units. VaR succeeded to draw a structured process for a better assessment of risk.

On the other hand, VaR was used to test the efficiency of the GARCH model; Engle (2001) estimates the $\mathrm{VaR}$ of a portfolio that exhibits $\mathrm{ARCH}$ effects using the GARCH $(1,1)$. While ARCH models assume equally weighted average of the squared residuals for a limited period, GARCH models assign decreasing weights to older observations. Such model is successful in forecasting the volatility of auto correlated returns. However, some practical extensions are needed such as GARCH $(p, q)$ that is found to be useful when applied with a very large set of data. Engle cites Nelson's Exponential GARCH (EGARCH) as being a good alternative when there is a need to integrate the sign in addition to the magnitude of news when forecasting volatility. Riposo and Bianca (2015) were the first to model the time-continuous ARCH (1)-M and GARCH $(1,1)-\mathrm{M}$ processes where the volatility has been constrained to take values into a bounded domain. The authors showed the uniqueness of the processes and derived the corresponding stationary probability functions. Vilasuso (2002) tested the predictive ability of an alternative model to $\mathrm{GARCH}$, which is the fractionally integrated GARCH (FIGARCH) that appears to be appropriate in incorporating long-lasting shocks.

Many approaches are used to measure VaR with numerous variations within each approach. The initial point starts with making assumptions about distributions for market risks. Then hypothetical portfolios can be built using historical data, variances and covariances across these risks or from Monte Carlo simulations. In this study, we compare the VaR estimates accuracy using the Delta-Normal and the Historical Simulation approaches.

The paper is organized as follows. Section 2 is a literature review of the CDS mechanism and the use of VaR. Section 3 presents the sample and reviews the methodology. Section 4 provides the main findings of the research and discusses the use of other alternatives for optimal accuracy. Section 5 communicates the concluding remarks.

\section{CDS Mechanism and the Use of VaR}

A credit default swap is a credit derivative contract. Simply stated, it is a swap particularly designed to transfer the credit exposure of fixed income products between two or three parties. In other words, it is an insurance contract written on the principal (notional value) of a bond for a fixed period of time where the buyer of the swap makes payments to the seller up until the maturity date. If the debt issuer defaults or faces a credit event such as a failure or change to pay the interest or principal, or financial restructuring, the insured receives the face value from the insurer (Berndt et al., 2007). If no default occurs, the insurance terminates with the contract expiration. The buyer pays periodical premiums, usually quarterly, to the seller over the lifetime of the contract, typically 5 years (Anson et al., 2004). The premiums are proportional to the notional principal of the contract. A CDS may involve municipal bonds, mortgage-backed securities (called Asset Backed Security (ABS)), emerging market bonds, or corporate bonds.

If the contract specifies physical settlement, the buyer has the right to sell to the seller of protection bonds issued by the reference entity and receive a cash payment equal to the notional value. If there is a cash settlement, a two-stage auction process is used to determine the midmarket value of the cheapest to deliverable bond several days after the credit event (Hull, 2012). Most contracts specify physical settlement.

The standard CDS contract is generated by the International Swap and Derivatives Association (ISDA), an association of major market participants. CDS are traded in over-the-counter markets and are therefore riskoriented. Trading CDS requires from the financial institution to have a line of credit which is linked to its overall financial situation and from the seller to post collateral. An exception for posting collateral is made for highly rated protection sellers. Collateral is marked-tomarket and are adjusted according to the change in the rating of the protection seller. On average, $90 \%$ of credit derivatives trades are subject to collateral agreement (ISDA, 2010). In the case of default, this corresponds to 
either the failure of the buyer to continue paying the premium which automatically terminates the contract or the failure of the seller on the date of a credit event when the claim is payable. Also, CDS contract could also be terminated if the collateral requirements are violated (Jarrow, 2010a). The losses due to the failure of the seller are much greater than those due to failure of the CDS buyer. Practically, the leverage involved in CDS trade can generate a widespread market downturn if default takes place.

According to the structural approach in the credit risk literature, selling a CDS isn't but buying the underlying reference bond concomitantly with shorting Treasuries with identical coupons, maturity and notional principal to that of the reference bond (Jarrow, 2010b). CDS are used for hedging and for speculation purposes. Buyers of protection are usually highly exposed to credit risk while speculators gamble on the credit quality of the reference entity. Therefore short positions are taken with positive expectations with regard to credit quality and long positions for negative view where buyers buy protection for small periodic fee and receive important payoff in case of defaults. A new family of harmonized CDS indices has been introduced in 2004: iTraxx in Europe and Asia and CDX in North America. This enhanced market transparency and market liquidity in the credit market.

The use of CDS to hedge credit risk is not examined in this study. The purpose is to compute the risk of holding CDS in the trading book. More specifically, we compare the VaR of three CDS portfolios in the US, Europe and Asia zones using both the historical simulation and the Delta-Normal approaches. The following gives details about the $\mathrm{VaR}$ and its computational methods.

In the late 1980s, Till Guildmann created the term VaR. As head of global research at JPMorgan, he was concerned about stable earnings but fluctuations in market values; the risk management group of the bank decided at that time that 'value risks' were more important than 'earnings risks' therefore paving the way to VaR (Jorion, 2007). By $1993 \mathrm{VaR}$ was established as an important risk measure. In 1994, JPMorgan developed the RiskMetrics, a simplified version of their own system, which included variances and covariances for a set of market variables (Morgan, 1997). RiskMetrics was used to measure the VaR by many financial institutions and nonfinancial corporations. In 1995, the Basel Committee issued a proposal to amend the 1988 Accord. It was implemented in 1998 and was based on VaR. It is the measure that regulators have chosen to set the capital requirements for market risk, credit risk and operational risk (BIS, 2005).

Specifically, VaR is an attempt to provide a single number that summarizes the total risk in a portfolio
(Hull, 2012). It describes the quantile of the projected distribution of gains and losses over the target horizon. Simply, it is the maximum loss not exceeded with a given probability defined as the confidence level, over a given period of time. VaR has become an essential tool for risk managers since it provides a quantitative measure of downside risk based on current positions. The objective is to provide a reasonably accurate estimate of risk at a reasonable cost (Jorion, 2007). This involves choosing a method that is most appropriate for the portfolio at hand. A variety of models are used to compute $\mathrm{VaR}$ with different variations within each model. The most commonly used models are the DeltaNormal or model-building approach, the historical simulation and the Monte Carlo simulation approach.

The Delta-Normal approach assumes a model for the joint distribution of changes in market variables and uses the historical data to estimate the model parameters. It is based on Harry Markowitz's work in portfolio theory. Therefore it fits to a portfolio consisting of long and short positions in stocks, bonds, commodities and other products. In this case, the change in the value of the portfolio is linearly dependant on the percentage changes in the prices of the assets comprising the portfolio. If daily returns are assumed to be multivariate normal, the probability distribution for the change in value of the portfolio over one day is also normal. Then, computing the VaR becomes straightforward. This approach is difficult to use for non-linear products because it does not take account of the gamma of the portfolio. In other words, when the positions can be represented by their delta exposures, the measurement of VaR is considerably simplified. This delta-normal approach is easily implemented because it involves a simple matrix multiplication. It is also fast even with a large number of assets because it replaces each position by its linear exposure and consequently it can be run in real time as positions change.

The historical simulation approach is a nonparametric method that makes no specific assumption about the distribution of risk factors. It consists of using the dayto-day changes in the values of the market variables that have been observed in the past in a direct way to estimate the probability distribution of the change in the value of the current portfolio between today and tomorrow. Each scenario is then drawn from the history of the studied observations (Jorion, 2007). This approach short circuits the need to estimate a covariance matrix which simplifies the calculation when portfolios are constituted of large number of assets and short sample periods (Naimy, 2013). What is needed is only the time series of the aggregate portfolio value. This method does not require distributional assumptions and therefore is robust and can capture gamma and vega risk. Historical simulation is also intuitive; users can go back in time and analyze the circumstances behind the VaR measure. 
Monte Carlo simulation method is a parametric method that generates random movements in risk factors from estimated parametric distributions. The parametric stochastic process is first specified together with some parameters such as risk and correlations which are derived from historical data. Then fictitious price paths are simulated for all the risk factors. At each horizon, the portfolio is marked to market as in the historical simulation method. Each of these simulated realizations is used to compile a distribution of returns, from which a VaR figure can be measured (Jorion, 2007). Monte Carlo approach method is a powerful method to compute VaR. It accounts for the instruments in the portfolio for nonlinear price exposure, vega risk and complex pricing models in addition to the flexibility in incorporating time variation in volatility and extreme scenarios.

\section{Sample and Methodology}

The selection of the approaches we will be using to measure and compare the VaR of CDS depends on those that will yield the most reliable estimate of $\mathrm{VaR}$ given the nature of the portfolios we are analyzing. In fact, the selection of the models depends on the inputs we are collecting. The three approaches will yield roughly the same values if the historical returns are normally distributed with consistent means and variances and are used to estimate the variance-covariance matrix.

Our analysis consists of comparing the VaRs of CDS of some selected highly rated banks and financial institutions in the US, Europe and Asia zones. Our sample covers the period March 2013-November 2015, a period of financial distress in the three zones. The purpose is to compare the VaR accuracy using different approaches with regard to percentage of outcomes falling within the VaR forecast. Overall we have nearly 12,000 observations. The data was retrieved from Bloomberg. Table 1 lists the selected banks and financial institutions in the three zones.

Several periods of market turmoil and an overall decline in CDS returns occurred during the sample period. Table 2-4 provide the associated descriptive statistics for the CDS monthly returns for the period January 2013-November 2015 in the three zones.

Going back to May 2005, after the downgrade of Ford and General Motors an upward jump in CDS premia took place. This market turbulence put an upward pressure on CDS premia for a limited period. Recently, a decline in risk premia was observed due to relatively low equity market volatility. Institutional investors increased their demand for higher yielding assets especially after the resulting collapse of stock prices in China.

Since the delta-normal approach and Monte Carlo simulations will yield roughly the same values if all of the inputs are assumed to be normally distributed, we test the normality distribution of returns in the three zones to choose between both approaches. Figure 1 shows Lilliefors Test for the three zones.

Based on the normality test results in Table 5 that indicates acceptable normality in the three zones, we will choose the Delta-Normal approach. In addition, given that the historical and Monte Carlo simulation approaches will converge if the distributions we use in the latter are entirely based upon historical data, we will opt for the historical simulation model which will provide good estimate of the VaR.

\section{Implementation of the Historical Simulation Method}

As previously mentioned, the historical simulation is a nonparametric method that consists of going back in time and implementing the history on the current positions. This model applies current weights to a time series of historical asset returns, that is:

$R_{p, k}=\sum_{i=1}^{N} w_{i, t} R_{i, k} \quad k=1, \ldots, t$

The weights $w_{\mathrm{t}}$ are kept at their currents values. This return does not represent an actual portfolio but rather reconstructs the history of a hypothetical portfolio using the current position. The approach is also called bootstrapping because it uses the actual distribution of recent historical data without replacement. Each scenario $k$ is drawn from the history of $t$ observations (Jorion, 2007). Then, hypothetical values for the risk factors are used and obtained from applying historical changes in prices to the current level of prices:

$S_{i, k}^{*}=S_{i, 0}+\Delta S_{i, k} \quad i=1, \ldots, N$

A new portfolio value $V_{p, k}^{*}$ is computed from the full set of hypothetical prices. This creates the hypothetical return corresponding to simulation $k$ :

$R_{p, k}=\frac{V_{k}^{*}-V_{0}}{V_{0}}$

$\mathrm{VaR}$ then is obtained from the entire distribution of hypothetical returns, where each scenario is assigned the same weight of $(1 / t)$.

In the three zones 500 scenarios were created and VaRs were then calculated for the period May 2013 through May 2015.

Figure 2 and 3 depict portfolios losses in Asia and Europe. US and Europe portfolios were following practically the same tempo. 
Normal and empirical cumulative distributions of European portfolio

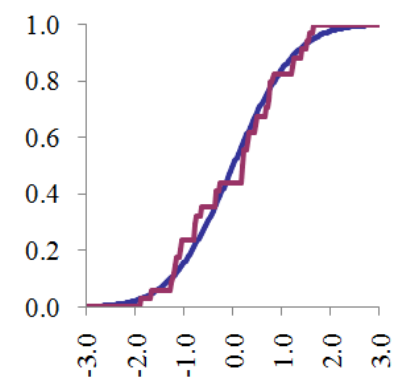

Normal and empirical cumulative distributions of Asian portfolio

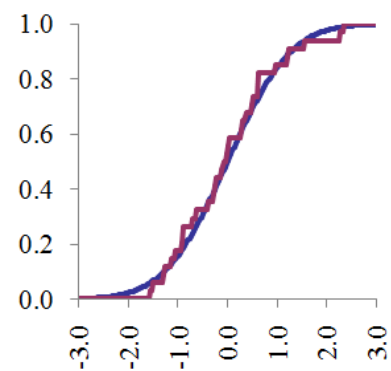

Normal and empirical cumulative distributions of US portfolio

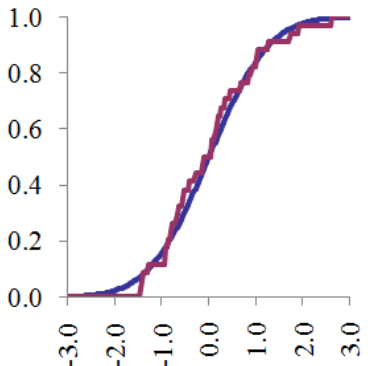

Fig. 1. Lilliefors normality test

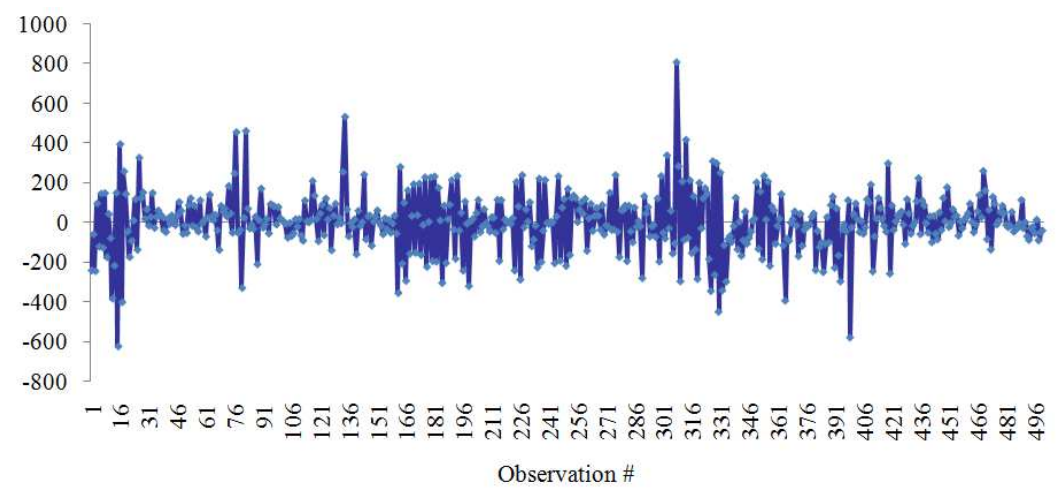

Fig. 2. Time series of portfolio losses Asia

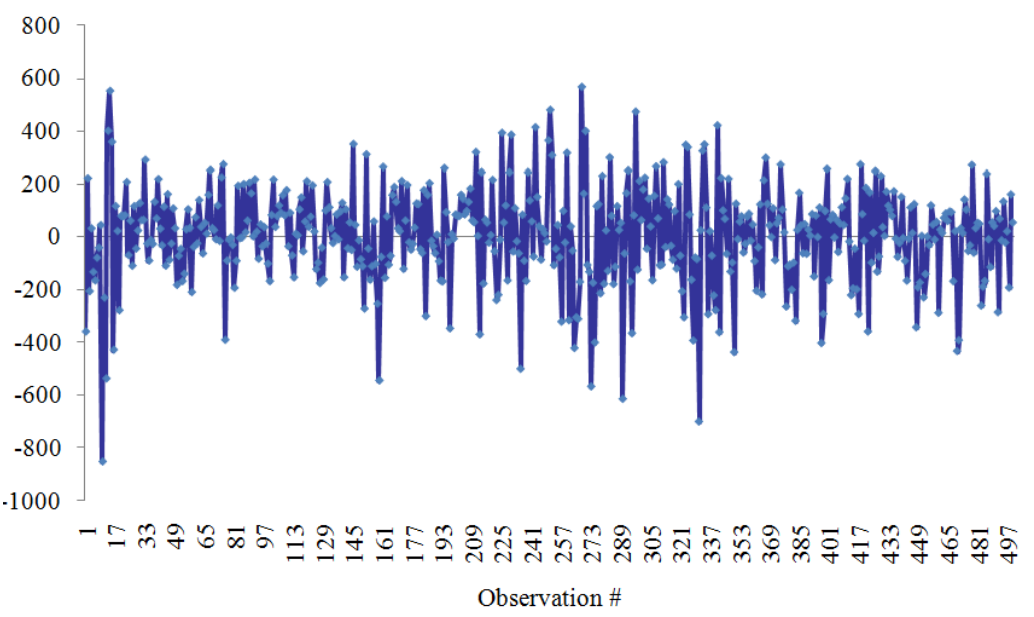

Fig. 3. Time series of portfolio losses Europe

Table 1. The sample

\begin{tabular}{lll}
\hline Europe & US & Asia \\
\hline UniCredit (UCGIM) & Bank of America (BOFA) & Bank of Tokyo (BOTM) \\
UBS Bank (UBS) & American Express Co (AXPRS) & Nomura Securities (NOMURAS) \\
Lloyds Bank (LLOY) & Citigroup Inc (CINC) & Mizuho Bank (MIZUHOB) \\
Royal Bank of Scotland (RBSPLC) & JP Morgan Chase and Co. (JPMCC) & Sumiyomo Mitsui Banking (SMBC) \\
Societe General (SOCGEN) & Wells Fargo and Co (CWF) \\
Deutsche Bank (DB) & Merrill Lynch and Co Inc (MER) & \\
Credit Suisse Group (CRDSUI) & Mack-Cali Reality (CLI) & \\
\hline
\end{tabular}


Viviane Y. Naimy / Journal of Mathematics and Statistics 2016, 12 (2): 99.106 DOI: 10.3844/jmssp.2016.99.106

Table 2. Descriptive statistics for the US

\begin{tabular}{|c|c|c|c|c|c|c|c|}
\hline & AXPRS (\%) & BOFA $(\%)$ & CINC (\%) & JPMCC (\%) & CWF (\%) & MER (\%) & CLI (\%) \\
\hline Mean & -0.99 & -0.55 & -0.16 & 0.28 & -0.35 & -0.65 & 1.01 \\
\hline Variance & 1.55 & 1.54 & 1.63 & 1.24 & 1.28 & 1.21 & 0.67 \\
\hline Std. Dev. & 12.45 & 12.40 & 12.76 & 11.12 & 11.30 & 11.02 & 8.19 \\
\hline Skewness & 36.45 & 53.10 & 106.22 & 11.93 & 21.74 & 126.57 & 19.59 \\
\hline Median & -4.57 & -2.03 & -1.48 & -0.86 & -1.72 & -0.94 & 0.35 \\
\hline Mode & -10.29 & -14.17 & -6.26 & 13.06 & -1.68 & -0.91 & 0.25 \\
\hline Minimum & -23.95 & -25.04 & -15.98 & -17.45 & -18.48 & -20.43 & -16.95 \\
\hline Maximum & 24.31 & 34.30 & 32.31 & 20.72 & 22.85 & 40.57 & 17.20 \\
\hline 1st Quartile & 10.03 & 10.21 & -9.14 & -7.27 & -10.04 & -5.68 & -4.00 \\
\hline 3rd Quartile & 5.63 & 8.08 & 7.16 & 10.83 & 8.48 & 5.07 & 4.95 \\
\hline
\end{tabular}

Table 3. Descriptive statistics for Asia

\begin{tabular}{|c|c|c|c|c|}
\hline & BOTM $(\%)$ & NOMURASE (\%) & MIZUHOB (\%) & SMBC (\%) \\
\hline Mean & 0.29 & -2.21 & -0.01 & 0.06 \\
\hline Variance & 2.29 & 1.05 & 1.48 & 1.52 \\
\hline Std. Dev. & 15.12 & 10.23 & 12.15 & 12.31 \\
\hline Skewness & 182.02 & 72.08 & 80.17 & 28.95 \\
\hline Median & -0.60 & -2.05 & -1.11 & -0.48 \\
\hline Mode & 2.37 & 2.95 & 2.60 & -0.42 \\
\hline Minimum & -20.88 & -20.32 & -21.14 & -26.14 \\
\hline Maximum & 49.69 & 26.24 & 31.88 & 26.97 \\
\hline 1st Quartile & -8.79 & -10.13 & -7.98 & -8.91 \\
\hline 3rd Quartile & 3.42 & 2.90 & 4.11 & 9.03 \\
\hline
\end{tabular}

Table 4. Descriptive statistics for Europe

\begin{tabular}{|c|c|c|c|c|c|c|c|}
\hline & UCGIM (\%) & UBS (\%) & LLOY (\%) & RBSPLC (\%) & SOCGEN (\%) & DB $(\%)$ & CRDSUI (\%) \\
\hline Mean & -1.17 & -1.48 & -2.60 & -2.18 & -1.57 & 0.65 & 0.23 \\
\hline Variance & 3.01 & 1.71 & 1.60 & 2.14 & 1.81 & 1.71 & 1.47 \\
\hline Std. Dev. & 17.35 & 13.10 & 12.67 & 14.63 & 13.47 & 13.08 & 12.11 \\
\hline Skewness & 10.22 & -4.39 & 27.71 & 55.09 & 10.25 & -9.93 & 4.32 \\
\hline Median & 1.15 & -3.23 & -3.24 & -5.18 & -0.60 & 3.01 & -2.59 \\
\hline Mode & -12.80 & 9.95 & -8.89 & -11.02 & 5.67 & 8.01 & -6.98 \\
\hline Minimum & -29.52 & -27.00 & -28.90 & -28.63 & -25.19 & -21.92 & -19.29 \\
\hline Maximum & 33.76 & 20.73 & 27.58 & 34.28 & 28.89 & 26.70 & 21.93 \\
\hline 1st Quartile & -14.84 & -10.71 & -10.23 & -12.86 & -11.57 & -10.57 & -8.00 \\
\hline 3rd Quartile & 9.37 & 9.90 & 4.50 & 7.23 & 8.62 & 10.86 & 10.47 \\
\hline
\end{tabular}

Table 5. Normality test results

\begin{tabular}{llll}
\hline Lilliefors test results & US & Europe & Asia \\
\hline Test Statistic & 0.0937 & 0.1286 & 0.0925 \\
CVal (15\% Sig. Level) & 0.1299 & 0.1299 & 0.1299 \\
CVal (10\% Sig. Level) & 0.1373 & 0.1373 & 0.1373 \\
CVal (5\% Sig. Level) & 0.1500 & 0.1500 & 0.1500 \\
CVal (2.5\% Sig. Level) & 0.1601 & 0.1601 & 0.1601 \\
CVal (1\% Sig. Level) & 0.2192 & 0.2192 & 0.2192 \\
\hline
\end{tabular}

Table 6 . VaR accuracy outcomes

\begin{tabular}{|c|c|c|c|c|c|c|}
\hline \multirow[b]{2}{*}{ Method } & \multicolumn{3}{|c|}{$95 \% \mathrm{VaR}$} & \multicolumn{3}{|c|}{$99 \% \mathrm{VaR}$} \\
\hline & US $(\%)$ & Europe $(\%)$ & Asia $(\%)$ & US $(\%)$ & Europe $(\%)$ & Asia $(\%)$ \\
\hline Delta-normal with equal weights over 501 days & 95.2 & 95.1 & 95.0 & 97.4 & 98.3 & 98 \\
\hline Historical simulation with equal weights over 501 days & 94.6 & 94.4 & 94.3 & 98.4 & 98.6 & 99 \\
\hline
\end{tabular}

Table 7. Delta-normal with exponential weights

\begin{tabular}{|c|c|c|c|c|c|c|}
\hline \multirow[b]{2}{*}{ Method } & \multicolumn{3}{|c|}{$95 \% \mathrm{VaR}$} & \multicolumn{3}{|c|}{$99 \% \mathrm{VaR}$} \\
\hline & US (\%) & Europe (\%) & Asia $(\%)$ & US (\%) & Europe $(\%)$ & Asia $(\%)$ \\
\hline Delta-Normal with exponential weights & & & & & & \\
\hline$\lambda=0.94$ & 94.7 & 94.6 & 94.9 & 97.2 & 98.1 & 98.0 \\
\hline$\lambda=0.99$ & 95.2 & 95.1 & 95.0 & 97.4 & 98.3 & 98.2 \\
\hline
\end{tabular}




\section{Implementation of Delta-Normal Method}

When the risk factors are jointly normally distributed and the positions can be represented by their delta exposures, the measurement of VaR becomes simple. Assuming $N$ the number of risk factors, we define $w_{i, t}$ as the exposures aggregated across all instruments for each risk factor $i$ and measured in currency units. Dividing these by the portfolio value $\mathrm{W}$, we get the portfolio weights $w_{i, t}$. The total rate of return of the portfolio is:

$$
R_{p, t+1}=\sum_{i=1}^{N} w_{i, t} R_{i, t+1}
$$

Weights are indexed by time to indicate that this is the current portfolio. Given that portfolios of jointly normal variables are themselves normally distributed, aggregation of risks becomes possible. Using matrix notations, the portfolio variance is:

$$
\sigma^{2}\left(R_{p, t+1}\right)=w_{t}^{\prime} \sum_{t+1} w_{t}
$$

$\Sigma_{t+1}$ is the forecast of the covariance matrix over the $\mathrm{VaR}$ horizon. Therefore, the portfolio $\mathrm{VaR}$ is:

$$
\mathrm{VAR}=\alpha \sqrt{x_{t}^{\prime} \sum_{t+1} x_{t}}=\alpha W \sqrt{w_{t}^{\prime} \sum_{t+1} w_{t}}
$$

where, $\alpha$ is the deviate corresponding to the confidence level for the normal distribution or for another parametric distribution.

\section{Findings and Discussion}

Table 6 compares the 1-day VaR numbers obtained from the two approaches for the three zones in terms of percentage of outcomes falling within the VaR forecast.

The above percentages are obtained by taking one minus the fraction of exception. At the $95 \%$ confidence level both approaches lead to an acceptable level of accuracy. At the 99\% confidence level, the Delta-Normal approach underestimates the $\mathrm{VaR}$ compared to the historical-simulation approach and both approaches are not finely calibrated except for Aisa zone under the historical simulation method. Results are not accurate using both approaches over 50 days, even at the $95 \%$ confidence level where all outputs fall below $95 \%$.

In fact the Delta-Normal VaR measures should be increased by about $13 \%$ to yield an accurate coverage this is due to the fat tails observed in the data, despite the acceptable Lilliefors test results. Another distribution can be chosen perhaps with a greater $\alpha$ to compensate for the proportion of outliers given that our position is characterized with linear risk profiles.

For a better accuracy, we run the Delta-Normal model using exponential weights for different values of $\lambda$. Table 7 shows that accuracy in the three zones did not improve much with the use of exponential weights. Almost same results compared to equal weights were observed for $\lambda=0.99$.

Obviously, in the Delta-Normal model, VaR attempts to capture the behavior of the CDS portfolio returns in the left tail (fat tails) which explains the underestimation of the VaR in the US and Europe portfolios. As we have seen, the underestimation increased for higher confidence levels. This approach is definitely not appropriate for nonlinear instruments because it is unable to capture asymmetries in the distribution. This approach looks relatively adequate for the three portfolios we are analyzing: Asymmetries are in fact washed away as predicted by the central limit theorem. Fortunately, the Historical-Simulation approach was accurate, may be by chance in our case, because only one sample path was used and it was relatively constant and homogeneous. If the window we used omits important events, the tails will not be well represented and vice versa, the sample may contain events that will not reappear in the future.

Other methods that we did not use in this study could be also suggested for better accuracy such as the Delta, Delta-gamma-delta, Delta-gamma-Monte Carlo, Grid Monte Carlo, or Full Monte Carlo approaches. Pritsker (1997) examines the tradeoff between speed and accuracy for a non-linear portfolio and found that the most accurate method is the full Monte Carlo which is very close to the true VaR.

\section{Conclusion}

Both approaches presented accuracy with regard to VaRs measures in the three zones. This was mainly due to the composition of our selected portfolios, to the distributions close to normal and to the selection of the period under study that witnessed relatively consistent events where the past represented fairly the immediate future. The historical simulation method was accurate because it accounted for fat tails especially that our window of estimation covered 500 days. This was reasonable to avoid inaccuracy and nonstationarity. The Delta-Normal approach was less accurate at the $99 \%$ confidence level due to the existence of those tails in the distribution of returns in the three CDS portfolios, however it was easy to implement. Even if Monte Carlo approach is the most powerful to compute VaR due to its flexibility to incorporate time variation in volatility and extreme scenarios, the nature of the distributions we are studying did not require the use of this method.

Our choice of methods won't be acceptable in the context of large and diversified portfolios even if the marked trend is toward the use of historical simulation methods. The Monte Carlo approach is the most complete however difficult to implement since it requires 
the use of a stochastic process for the risk factors from which various sample paths are simulated. It can incorporate nonlinear positions and nonnormal distributions but the price to pay for such flexibility is high.

\section{Acknowledgement}

The author is very grateful for the constructive and helpful comments of Professor Carlo Bienca.

\section{Ethics}

This article is original and contains unpublished material. The corresponding author confirms that all of the other authors have read and approved the manuscript and no ethical issues involved.

\section{References}

Anson, M., F. Fabozzi, M. Choudhry and R. Chen, 2004. Credit Derivatives: Instruments, Applications and Pricing. 1st Esn., John Wiley and Sons, Inc., New Jersey, ISBN-10: 0471652385, pp: 288.

BIS, 2005. Basel II: International convergence of capital measurment and capital standards: A revised framework. Bank of International Settlements.

Berndt, A., R. Jarrow and C. Kang, 2007. Restructuring risk in credit default swaps: An empirical analysis. Stochastic Processes Applic., 117: 1724-1749. DOI: $10.1016 /$ j.spa.2007.01.013

Duffie, D., A. Li and T. Lubke, 2010. Policy perspectives on OTC derivatives market infrastructure. Federal Reserve Bank of New York Staff Reports.
Engle, R., 2001. GARCH 101: The use of ARCH/GARCH models in applied econometrics. J. Econom. Perspectives, 15: 157-168.

Hull, J., 2012. Risk Management and Financial Institutions. 3rd Edn., John Wiley and Sons, Hoboken, ISBN-10: 1118282914, pp: 672.

ISDA, 2010. Margin survey preliminary results. International Swaps and Derivatives Association.

Morgan, J.P., 1997. Credit metrics technical document.

Jarrow, R., 2010a. The Economics of Credit default Swaps (CDS). Johnson School Research Paper Series.

Jarrow, R., 2010b. A simple robust model for cat bond valuation. Finance Res. Lett., 7: 72-79. DOI: $10.1016 /$ j.frl.2010.02.005

Jorion, P., 2007. Value at Risk: The New Benchmark for Managing Financial Risk. 3rd Edn., McGraw Hill Professional, New York, ISBN-10: 0071736921, pp: 600.

Naimy, V., 2013. Parameterization of $\operatorname{GARCH}(1,1)$ for Paris stock market. Am. J. Math. Statist., 3: 357-361. DOI: 10.5923/j.ajms.20130306.09

Pritsker, M., 1997. Evaluating value at risk methodologies: Accuracy versus computational time. J. Financial Services Res., 12: 201-242. DOI: $10.1023 / \mathrm{A}: 1007978820465$

Riposo, J. and C. Bianca, 2015. On volatility variation in $\operatorname{ARCH}(1)$ and $\operatorname{GARCH}(1,1)$ continuous limits. Nonlinear Studies, 22: 359-371.

Vilasuso, J., 2002. Forecasting exchange rate volatility. Econom. Lett., 76: 59-64. DOI: $10.1016 / \mathrm{S} 0165-1765(02) 00036-8$ 